\title{
Treatment of Legal Sentences Including Itemized and Referential Expressions - Towards Translation into Logical Forms -
}

\author{
Yusuke Kimura, Makoto Nakamura, and Akira Shimazu \\ School of Information Science \\ Japan Advanced Institute of Science and Technology \\ 1-1, Asahidai, Nomi, Ishikawa, 923-1292, Japan \\ \{mnakamur, shimazu\}@jaist.ac.jp
}

\begin{abstract}
This paper proposes a framework for analyzing legal sentences including itemized or referential expressions. Thus far, we have developed a system for translating legal documents into logical formulae. Although our system basically converts words and phrases in a target sentence into predicates in a logical formula, it generates some useless predicates for itemized and referential expressions. We propose a front end system which substitutes corresponding referent phrases for these expressions. Thus, the proposed system generates a meaningful text with high readability, which can be input into our translation system. We examine our system with actual data of legal documents. As a result, the system was $73.1 \%$ accurate in terms of removing itemized expressions in a closed test, and $51.4 \%$ accurate in an open test.
\end{abstract}

\section{Introduction}

A new research field called Legal Engineering was proposed in the 21st Century COE Program, Verifiable and Evolvable e-Society [1,2]. Legal Engineering serves for computer-aided examination and verification of whether a law has been established appropriately according to its purpose, whether there are logical contradictions or problems in the document per se, whether the law is consistent with related laws, and whether its revisions have been modified, added, and deleted consistently. One approach to verifying law sentences is to convert law sentences into logical or formal expressions and to verify them based on inference [3].

This paper reports our ongoing research effort to build up a system for automatically converting legal documents into logical forms. The system analyzes law sentences, determines logical structures, and then generates logical expressions. Thus far, we have shown our system provides high accuracy in terms of generating logical predicates corresponding to words and their semantic relations [4]. However, some predicates generated concerned with itemization and reference were meaningless, because predicates converted from words and phrases, such as "the items below," "Article 5," and so on are not intrinsic to a logical representation of the sentence. These words should be replaced with appropriate phrases 
before the process of translation. Accordingly, our purpose in this paper is to propose a method to rewrite legal sentences including itemization or reference into an independent, plain sentence. We consider that this system is useful not only for the front end processor of our main system for translating legal sentences into logical forms, but also for assistance for reading legal documents.

In this paper, we introduce our current system and its problems in Section 2. In Section 3 we show analysis of law sentences including itemization or reference, and we propose a method to rewrite the law sentences into plain sentences in Section 4. We also examine our new method and report its results in Section 5. Finally, we conclude and describe our future work in Section 6 .

\section{The Current System and Problems}

In this section, we describe our current system for translating legal documents into logical forms, and its problems. We call our system WILDCATS ${ }^{1}$.

\section{$2.1 \quad$ Work Related to Wildcats}

Acquisition of knowledge bases by automatically reading natural language texts has widely been studied. Because the definition of semantic representation differs depending on what the language processing systems deal with, a few systems try to generate logical formulae based on first order predicate logic [5]. A study of knowledge acquisition by Mulkar et al. [6,7] is one of those systems. They extracted well-defined logical formulae from textbooks of biology and chemistry. As a result, their model succeeded in solving some high school AP exam questions. Legal documents are different from the textbooks in that they are described with characteristic expressions in order to avoid ambiguous description. Therefore, we take into account analysis of the expressions based on the linguistic investigation.

In most cases, a law sentence in Japanese Law consists of a law requisite part and a law effectuation part, which designate its legal logical structure $[8$, 9]. Structure of a sentence in terms of these parts is shown in Fig. 1. The law requisite part is further divided into a subject part and a condition part, and the law effectuation part is divided into an object, content, and provision part.

Dividing a sentence into these two parts in the pre-processing stage makes the main procedure more efficient and accurate. Nagai et al. [9] proposed an acquisition model for this structure from Japanese law sentences. Dealing with strict linguistic constraints of law sentences, their model succeeded in acquiring the structures at fairly high accuracy using a simple method, which specifies the surface forms of law sentences. Our approach is different from theirs in that we consider some semantic analyses in order to represent logical formulae.

\footnotetext{
${ }^{1}$ WILDCATS is an abbreviation of "Wildcats' Is a Legal Domain Controller As a Translation System."
} 


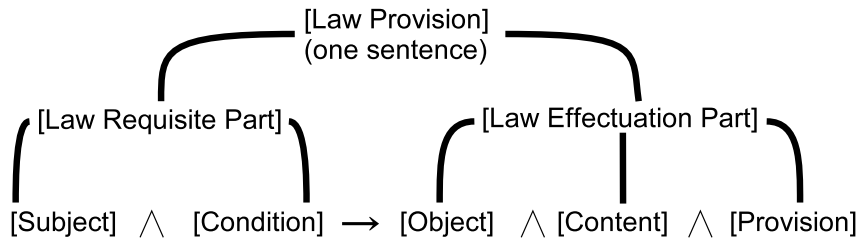

Fig. 1. Structure of requisition and effectuation [8]

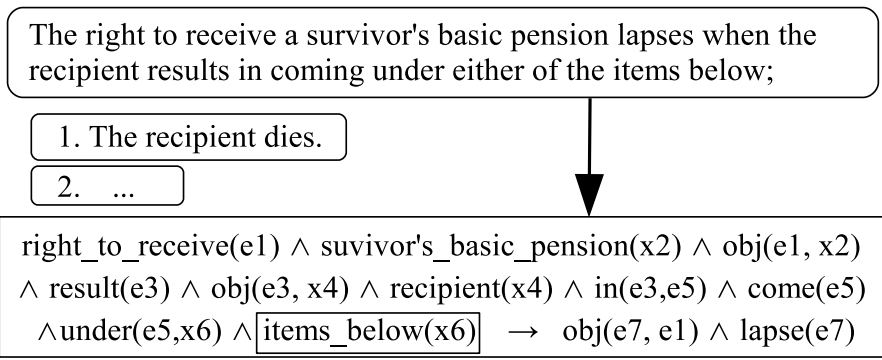

Fig. 2. Converting a law sentence including a reference phrase

\subsection{Wildcats}

Here, we explain an outline of our current system. The following list is the procedure for one sentence. We repeat it when we process a set of sentences.

1. Analyzing morphology by JUMAN and parsing a target sentence by KNP.

2. Splitting the sentence based on the characteristic structure of a law sentence.

3. Assignment of modal operators with the cue of auxiliary verbs.

4. Making one paraphrase of multiple similar expressions for unified expression.

5. Analyzing clauses and noun phrases using a case frame dictionary.

6. Assigning variables and logical predicates. We assign verb phrases and sahennouns $^{2}$ to a logical predicate and an event variable, $e_{i}$, and other content words to $x_{j}$, which represents an argument of a logical predicate.

7. Building a logical formula based on fragments of logical connectives, modal operators, and predicates.

The procedure is roughly divided into two parts. One is to make the outside frame of the logical form (Step 1 to 3 and 7), which corresponds to the legal logical structure shown in Fig. 1. The other (Step 4 to 6 ) is for the inside frame. We assign noun phrases to bound variables and predicates using a case frame dictionary. We show an example of input and output in Fig. 2.

\footnotetext{
${ }^{2}$ A sahen-noun is a noun which can become a verb with the suffix -suru.
} 
Table 1. Typical reference phrases in National Pension Law

\begin{tabular}{|c|c|}
\hline Reference phrases & Frequency \\
\hline X-ni kitei-suru $\mathrm{Y}_{\text {noun }} \quad$ (X に規定する $\mathrm{Y}$ (名詞)) & 103 \\
\hline $\mathrm{Y}_{\text {noun }}$ which is enacted in $\mathrm{X}$ & \\
\hline $\begin{array}{l}\mathrm{X} \text {-no kitei-niyoru } \mathrm{Y}_{\text {noun }}(\mathrm{X} \text { の規定による } \mathrm{Y}(\text { 名詞 })) \\
\mathrm{Y}_{\text {noun }} \text { which is enacted in } \mathrm{X}\end{array}$ & 71 \\
\hline $\begin{array}{ll}\mathrm{X} \text {-no kitei-niyori } \mathrm{Y}_{\text {verb }} & \text { (X の規定により } \mathrm{Y} \text { (動詞)) } \\
\mathrm{Y}_{\text {verb }} \text { as enacted in } \mathrm{X} & \end{array}$ & 109 \\
\hline
\end{tabular}

\subsection{Problems of Wildcats}

When our system converts a law sentence including referential phrases, they are not interpreted correctly. For example, in Fig. 2, the enclosed predicate "items_below $(\mathrm{x} 4)$ " is useless. This is because the generated predicates lack information which must be referred. These phrases should be replaced with appropriate phrases in the items before the process of translation into logical forms. Therefore, substituting corresponding referent phrases for these expressions appropriately, our proposed system in this paper generates a meaningful text with high readability, and then the generated text can be input to the translation system. For example, the system should process the following instead of the input sentences in Fig. 2; "The right to receive a survivor's basic pension lapses when the recipient dies."

The scope of the study in this paper is restricted to itemized and referential expressions. Therefore, in the following sections we show analysis of law sentences and explain our methodology, which is based on the previous study by Ogawa et al. [10], who proposed a method for rewriting texts using regular expressions in order to consolidate legal sentences and amendment sentences.

\section{Analysis of Law Sentences}

\subsection{Analysis of Reference in Law Sentences}

There are reference phrases in law sentences, for example "X -ni kitei-suru Y (Y which is enacted in X)." In National Pension Law, typical reference phrases are shown in Table 1.

In these phrases, $\mathrm{X}$ acts as a pointer to another law sentence. We show some examples of reference phrases found in National Pension Law, as follows:

- Item $a$, Paragraph $b$, Article $c$ (absolute pointer)

- the previous paragraph (relative pointer)

- Paragraph $b$ in the previous article (combination)

- the same article

- the previous $n$ articles

- a proviso in the previous article 


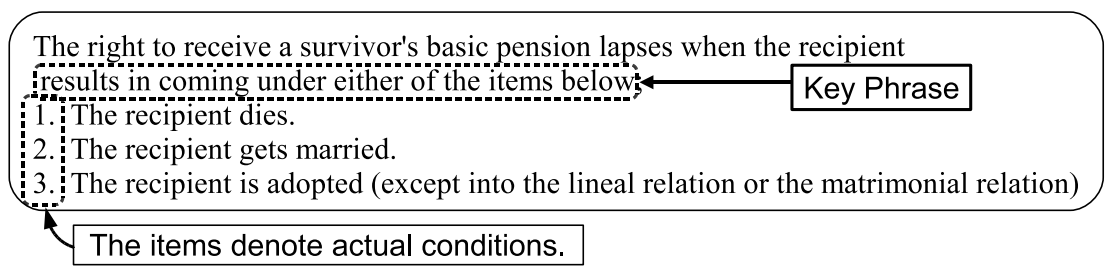

Fig. 3. Itemization of conditions in the law requisite part

- this law (self-reference)

We also examined frequency in the use of the noun $\mathrm{Y}$ in the sentence indicated by a pointer $\mathrm{X}$, because we consider that the noun $\mathrm{Y}$ is explained in detail in the sentence indicated by X. For example, a phrase "the postponement of issuance which is enacted in Paragraph 1, Article 28" implies that we can find a more detailed phrase "postponement of issuance of the old age basic pension" in Paragraph 1, Article 28. Therefore, we regarded the sentence indicated by X as an explanation of the noun $\mathrm{Y}$.

We targeted the phrase "X-ni kitei-suru Y ( $\mathrm{Y}$ which is enacted in $\mathrm{X}$ )," as it appears most frequently in National Pension Law. A pointer X indicates another law document in 21 cases out of 103 , and we examined the remaining 82 cases. As a result, in 49 cases the noun $\mathrm{Y}$ appears only once in the sentence indicated by $\mathrm{X}$, and twice or more in 24 cases, while the sentence indicated by $\mathrm{X}$ does not contain the noun $\mathrm{Y}$ in only 9 cases. Therefore, it is easy to find the part of the explanation, which is located near the noun Y. With this idea, we consider a method to extract an explanation from a sentence indicated by X in Section 4.1.

\subsection{Analysis of Itemization in Law Sentences}

Some law sentences include itemization of conditions in the law requisite part, an example of which is shown in Fig. 3. The enclosed phrase should be replaced with one of the items denoting actual conditions. When one or more conditions are satisfied, the description in the law effectuation part becomes effective. We found 34 sentences of such a style in National Pension Law. Therefore, we considered a method to embed itemized conditions instead of cue phrases of itemization.

We defined Key Phrases, which always appear in sentences before itemization $^{3}$. As we analyzed sentences from all 215 articles of the National Pension Law, the set of Key Phrases can be expressed as a regular expression, the diagram of which is shown in Fig. 4. For example, the phrase "Tsugi no kaku gou ni gaitou suru ni itatta," meaning "to result in coming under either of the items below ${ }^{4}$," which is derived from the generative rule in Fig. 4, is regarded as a Key Phrase.

\footnotetext{
3 There may be a proviso between the sentence and itemization

${ }^{4}$ If we do not care about word-to-word translation for the Japanese law sentence, the following phrase is more appropriate; "to be included in one of the following cases."
} 


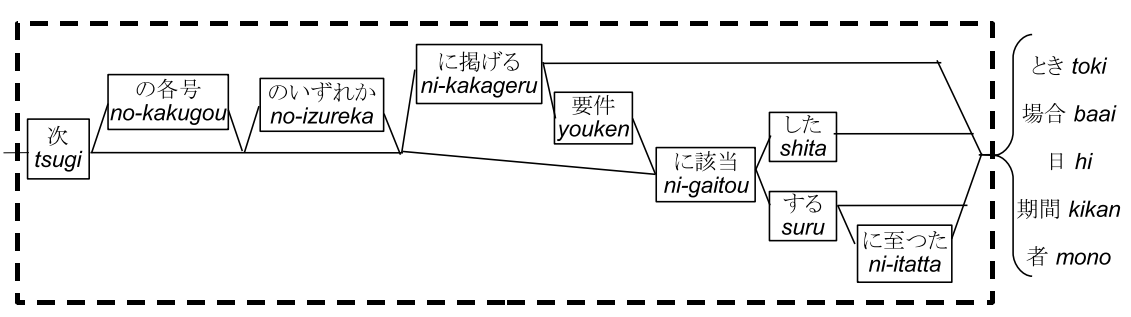

Fig. 4. Key phrases for itemization

Table 2. Frequency of Key Phrases

\begin{tabular}{lr}
\multicolumn{2}{c}{ KP: Key Phrase) } \\
\hline Format of KPs / Frequency \\
\hline KP + toki (とき) & 9 \\
$\mathbf{K P}+$ baai (場合) & 9 \\
$\mathbf{K P}+$ mono (者) & 6 \\
$\mathbf{K P}+$ hi $($ 日) & 3 \\
$\mathbf{K P}+$ kikan (期間) & 1 \\
$\mathbf{K P}+$ youken (要件) & 1 \\
$\mathbf{K P}+$ a noun & 5 \\
\hline Total & 34 \\
\hline
\end{tabular}

Table 3. Frequency of Condition Items

\begin{tabular}{lr} 
CI: Condition Items \\
\hline Format of CIs / Frequency \\
\hline CI + toki (とき) & 106 \\
CI + koto (こと) & 4 \\
CI + mono (もの) & 3 \\
CI + mono (者) & 2 \\
CI + a noun & 9 \\
\hline Total & 124 \\
\hline
\end{tabular}

Itemized condition sentences appear next to sentences which contain Key Phrases. The last words of these sentences are "Toki (time)," "Mono (person)," and so on. In this paper, we call these sentences excluding the last words Condition Items. Key Phrases and Condition Items appearing in National Pension Law are shown in Table 2 and Table 3, respectively.

We will describe a method to remove itemization using Key Phrases and Condition Items in Section 4.2.

\section{Method for Substituting Referent Phrases}

\subsection{Extracting an Explanation from Referent}

As was mentioned in Section 3.1, we show a method to extract a detailed explanation of a reference phrase, such as "X-ni kitei-suru $\mathrm{Y}$ (Y which is enacted in $\mathrm{X})$," from a referent sentence. The procedure is shown as follows;

1. Identifying a reference expression

2. Searching for the same words in the reference expression and the referent sentence

3. Syntactic analysis of the referent sentence and extraction of supplements

In the first step, if the sentence includes one of the phrases in Table 1, the system recognizes the phrase as a reference expression. We show an example 


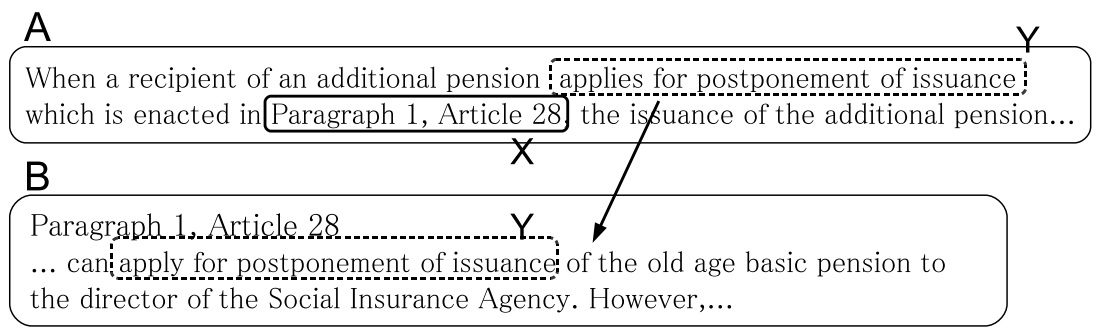

Fig. 5. (A) a reference expression, and (B) a referent sentence

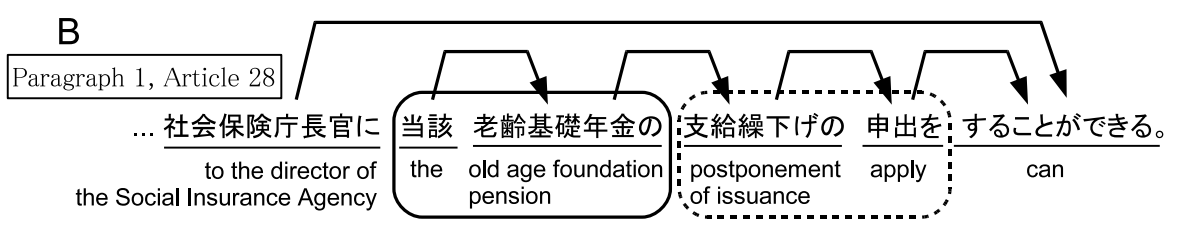

Fig. 6. The dependency tree of the referent sentence

of a reference expression in Fig. 5-A. The phrase "which is enacted in" is the reference phrase, and the referent sentence is shown in Paragraph 1, Article 28.

In the next step, the system searches for a phrase in the referent sentence, which is matched with the noun phrase corresponding to $\mathrm{Y}$ described in the reference sentence. A difficult thing is to determine the region of words as an identified phrase. The system recognizes the longest matched words as the noun phrase Y. In Fig. 5, the system extracted a phrase corresponding to Y as "apply for postponement of issuance." 5

Finally, the system analyzes the referent sentence with the Japanese morphological analyzer, JUMAN, and Japanese dependency analyzer, KNP. We regard elements which modify $\mathrm{Y}$ in the dependency tree as supplements for the word Y. Then, we replace the phrase "X-ni kitei-suru" with the supplements for the word Y. In this example shown in Fig. 6, "which is enacted in Paragraph 1, Article 28" is replaced with "of the old age basic pension."

\subsection{Removing itemization}

In Section 3.2, we defined Key Phrases as cue phrases that always appear with itemization, like "tsugi-no kaku gou no izureka ni gaitou-suru ((something) to which either of the following items is applicable)," and we search for itemization with it. If a Key Phrase is found, we regard the following items as Condition Items, and replace the Key Phrase with one of the Condition Items for each. Then

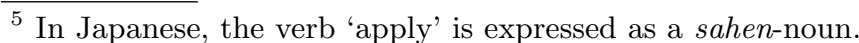




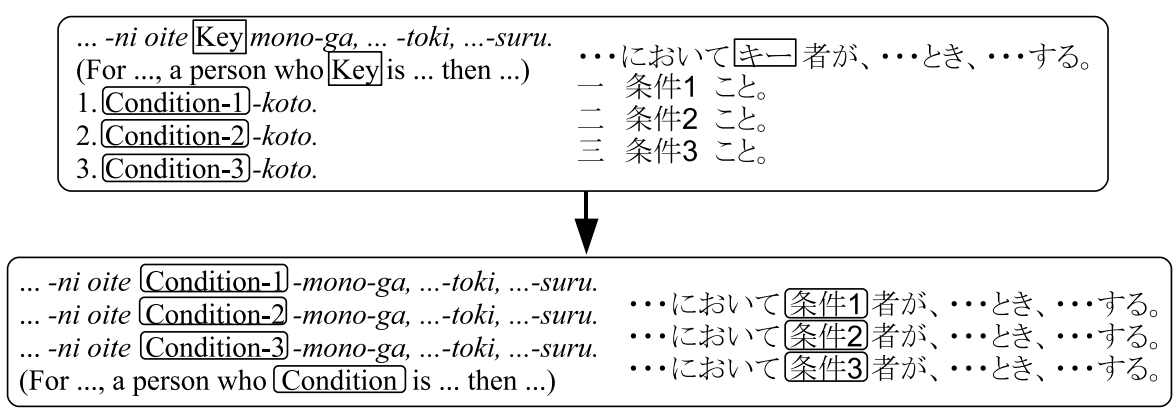

Fig. 7. Removing itemization

(a) Input

The right to receive a survivor's basic pension lapses

when the recipient results in coming under either of the items below;

1. The recipient dies.

2. The recipient gets married:

\section{(b) Output}

- The right to receive a survivor's basic pension lapses when the recipient dies.

- The right to receive a survivor's basic pension lapses when the recipient gets married

Fig. 8. An example of removing itemization

we have sentences which are understandable separately ${ }^{6}$, as shown in Fig. 7. We show an example of the pair of input and output in Fig. 8.

\section{Experiments and Results}

\subsection{Reference expressions in National Pension Law}

We tested our system on reference phrases "X-ni kitei-suru $\mathrm{Y}$ ( $\mathrm{Y}$ which is enacted in X)" in National Pension Law. The result is shown in Table 4. The system derived correct information from 41.5 percent of reference phrases in National Pension Law. For 20.8 percent of reference expressions, generated sentences were ungrammatical or not enough, since some necessary words or phrases were not expressed in output sentences. For example, some referent sentences contain a number of reference expressions. Even though all of them are an explanation of the reference expression, the system extracts only the one of them which appears first in the sentence. We judged the result to be partially extracted.

\footnotetext{
${ }^{6}$ Even though the converted logical formulae are repetitive, there is no problem as long as the system gives the same logical predicates and variables to the repetitive phrases.
} 
Table 4. Result for the reference expression Table 5. Result for identifying itemization "X-ni kitei-suru Y"

\begin{tabular}{l|r|r}
\hline & Sentence & $\%$ \\
\hline Extracted correctly & 22 & $41.5 \%$ \\
Extracted partially & 11 & $20.8 \%$ \\
Extracted nothing & 20 & $37.7 \%$ \\
\hline Total & 53 & $100 \%$ \\
\hline
\end{tabular}

\begin{tabular}{l|r|r}
\hline Identifying & Itemization & Conditions \\
& Frequency & \\
\hline Succeeded & 33 & 119 \\
Failed & 1 & 5 \\
\hline Total & 34 & 124 \\
\hline \hline Misidentify & 1 & 2 \\
\hline
\end{tabular}

Table 6. Result for removing itemization

\begin{tabular}{l|rr|rr} 
& National Pension Law & Income Tax Law & \\
\hline & conditions & $\%$ & conditions & $\%$ \\
\hline Succeeded & 87 & $73.1 \%$ & 219 & $51.4 \%$ \\
Wrong sentence & 21 & $17.7 \%$ & 123 & $28.9 \%$ \\
Error & 11 & $9.2 \%$ & 84 & $19.7 \%$ \\
\hline Total & 119 & $100 \%$ & 426 & $100 \%$ \\
\hline
\end{tabular}

\subsection{Experiment for Itemization}

We tested our system on itemization in National Pension Law. From the point of view of identifying itemization, our system found most itemization structures, shown in Table 5. The result of removing itemization is shown on the left hand side of Table 6 .

All of the errors are items which denote a combination of a Condition Item and an object part in the law effectuation part. In other words, the objects of these sentences change depending on the Condition Items.

We also inspected the system with Income Tax Law as an open test, shown on the right hand side of Table 6 . A little more than half of the sentences were processed well, there seems to be some difference in notation between National Pension Law and Income Tax Law. Particularly, we found the increase of itemization consisting of a combination of a Condition Item and an object part. Results will be improved after an analysis of the mistakes.

\section{Conclusion}

In this paper we proposed a method to rewrite legal sentences including itemization or reference into independent, plain sentences. In the experiments, we showed that the system successfully extracted itemized expressions with some exceptions. For referential expressions, focusing on a referential phrase "X-ni kitei-suru $Y$ " in National Pension Law, we showed the system worked well for extracting reference expressions. We consider that the system is useful not only for the front end of our main system, Wildcats, but also for assistance in reading legal documents.

Some tasks still remain in our future work: (1) As was shown in Section 5 , our system failed for some sentences. We can deal with some of the failures 
easily. (2) We can improve this system by introducing a method for measuring readability of the output sentences. (3) We will test our main system, Wildcats, using the proposed model as the front end system.

Acknowledgment This research was partly supported by the 21st Century COE Program 'Verifiable and Evolvable e-Society' and Grant-in-Aid for Scientific Research (19650028). We would like to give special thanks to Yoko Shibata, Yoshiko Oyama, and Akiko Hayashi, who analyzed law sentences and their logical representations.

\section{References}

1. Katayama, T.: The current status of the art of the 21st COE programs in the information sciences field (2): Verifiable and evolvable e-society - realization of trustworthy e-society by computer science - (in Japanese). IPSJ (Information Processing Society of Japan) Journal 46 (2005) 515-521

2. Katayama, T.: Legal engineering - an engineering approach to laws in e-society age -. In: Proc. of the 1st Intl. Workshop on JURISIN. (2007)

3. Hagiwara, S., Tojo, S.: Stable legal knowledge with regard to contradictory arguments. In: AIA'06: Proceedings of the 24th IASTED international conference on Artificial intelligence and applications, Anaheim, CA, USA, ACTA Press (2006) 323-328

4. Nakamura, M., Nobuoka, S., Shimazu, A.: Towards translation of legal sentences into logical forms. In Satoh, K., Inokuchi, A., Nagao, K., Kawamura, T., eds.: New Frontiers in Artificial Intelligence: JSAI 2007 Conference and Workshops, Miyazaki, Japan, June 18-22, 2007, Revised Selected Papers. Volume 4914 of Lecture Notes in Computer Science., Springer (2008) 349-362

5. Hobbs, J.R., Stickel, M., Martin, P., Edwards, D.: Interpretation as abduction. In: Proceedings of the 26th annual meeting on Association for Computational Linguistics, Morristown, NJ, USA, Association for Computational Linguistics (1988) 95-103

6. Mulkar, R., Hobbs, J.R., Hovy, E.: Learning from reading syntactically complex biology texts. In: Proceedings of the 8th International Symposium on Logical Formalizations of Commonsense Reasoning, part of the AAAI Spring Symposium Series. (2007)

7. Mulkar, R., Hobbs, J.R., Hovy, E., Chalupsky, H., Lin, C.Y.: Learning by reading: Two experiments. In: Proceedings of IJCAI 2007 workshop on Knowledge and Reasoning for Answering Questions. (2007)

8. Tanaka, K., Kawazoe, I., Narita, H.: Standard structure of legal provisions - for the legal knowledge processing by natural language - (in Japanese). In: IPSJ Research Report on Natural Language Processing. (1993) 79-86

9. Nagai, H., Nakamura, T., Nomura, H.: Skeleton structure acquisition of Japanese law sentences based on linguistic characteristics. In: Proc. of NLPRS'95, Vol 1. (1995) 143-148

10. Ogawa, Y., Inagaki, S., Toyama, K.: Automatic consolidation of Japanese statutes based on formalization of amendment sentences. In Satoh, K., Inokuchi, A., Nagao, K., Kawamura, T., eds.: New Frontiers in Artificial Intelligence: JSAI 2007 Conference and Workshops, Miyazaki, Japan, June 18-22, 2007, Revised Selected Papers. Volume 4914 of Lecture Notes in Computer Science., Springer (2008) 349-362 